\title{
Conflits Fonciers Et Problématique De Développement Rural Dans La Sous-Préfecture De Mongo, Région Du Guéra (Tchad)
}

\author{
Moussa Alladjaba \\ Chercheur en Géographie, Université de Dschang, Cameroun \\ Hervé Tchekote \\ Géographe, Enseignant-Chercheur, Chargé de cours au Département de \\ géographie, Université de Dschang, Cameroun
}

Doi:10.19044/esj.2018.v14n32p213 URL:http://dx.doi.org/10.19044/esj.2018.v14n32p213

\begin{abstract}
Located in the Guéra region in central Chad, the Mongo Sub-Prefecture is the scene of recurring conflicts related to the occupation and exploitation of the land. While the phenomenon is neither new nor specific to this part of the country, the scale it has taken in recent years makes it a worrying subject. This land conflicts are driven by a multitude of actors with traditional authorities, agricultural producers and the urban elite at the center. They are fuelled by the fact that the legal land tenure system, set up first by the colonial authority and then renewed by the Chadian authorities, has never been a reality in rural areas. To conduct the study the questionnaire survey was conducted from a sample of 106 households. Subsequently, semi-structured interviews supplemented the information collected through the questionnaires. The results reveal not only a pluralism of norms whose contradiction in implementation is inevitably conflicting, but also and above all the actors' play around rural land, with a multitude of conflicts whose impact is strongly felt by populations, both in terms of agricultural or pastoral production, and in terms of trade and social cohesion. At the end of the study, it turns out that the population largely ignores the laws governing the land. The result is a pluralism of norms, but the customary rules are preponderant. This determines several modes of access and use of the land.
\end{abstract}

Keywords: Land conflict, land system, impact, Guéra, Mongo sub-division, Chad

Résumé

Située dans la région du Guéra au centre du Tchad, la Sous-préfecture de Mongo est le théâtre des conflits récurrents liés à l'occupation et à 
l'exploitation de la terre. Certes, le phénomène n'est ni nouveau, ni spécifique à cette partie du pays, mais l'ampleur qu'il prend ces dernières années en fait un sujet préoccupant. Ces conflits fonciers sont animés par une multitude d'acteurs avec au centre les autorités traditionnelles, les producteurs agricoles et l'élite urbaine. Ils sont nourris par le fait que le système foncier légal, mis en place d'abord par l'autorité coloniale, puis reconduit par les autorités du Tchad indépendant n'a jamais été une réalité en milieu rural. Pour réaliser l'étude l'enquête par questionnaire a été menée auprès d'un échantillon de 106 ménages. Ensuite, des entretiens semi-structurés ont permis de compléter les informations recueillies au moyen des questionnaires. Les résultats révèlent non seulement un pluralisme des normes dont la contradiction dans la mise en œuvre est inéluctablement conflictogène, mais fait intervenir aussi et surtout des jeux d'acteurs autour du foncier rural, avec une multitude de conflits dont l'impact est durement ressenti par les populations, tant en termes de production agricole ou pastorale, qu'en termes d'échanges et de cohésion sociale. Au terme de l'étude, il se révèle que les populations méconnaissent largement les lois régissant le foncier. Il en résulte un pluralisme des normes, mais les règles coutumières sont prépondérantes. Ce qui détermine plusieurs modes d'accès et d'usage de la terre.

Mots-clés : conflit foncier- système foncier- impact, Guéra, Sous-préfecture de Mongo, Tchad.

\section{Introduction}

De nos jours, l'accès à la terre fait l'objet d'une compétition permanente entre de différents usagers. Dans ce contexte, la question foncière se retrouve au centre de toutes les stratégies en matière de sécurité alimentaire, de préservation de l'environnement et de gestion des ressources naturelles. Cependant, l'enjeu d'une gestion apaisée de cette ressource, tant en milieu rural que dans les zones urbaines, constitue un important facteur de stabilisation de cohésion sociale et de développement.

$\mathrm{Au}$ Tchad comme dans beaucoup de pays d'Afrique Subsaharienne, le problème foncier se pose en termes d'accès à la terre, mais aussi en termes de sécurisation des droits des différents usagers. Le problème majeur auquel l'Etat est confronté est celui de l'inapplicabilité des textes et des politiques (Houdeingar, 2009). En effet, bien que le législateur ait posé sans équivoque, dès le départ, le principe de la domanialité des terres, l'État peine à imposer son emprise sur le foncier, surtout en milieu rural. D'ailleurs, la loi n'a pas systématiquement aboli les droits coutumiers (Le Roy, 1995) et laisse cohabiter un dualisme de normes. Ainsi, ce dualisme, voire ce pluralisme des normes « rend problématique l'application de la loi et la puissance publique hésite souvent entre coercition et négociation avec les pouvoirs locaux en 
matière de régulation foncière » (Le Roy, 1995). Le droit coutumier occupe ainsi une place centrale, c'est-à-dire celle qui est laissée libre par l'ineffectivité du droit officiel (Durand-Lasserve et Le Roy, 2012). Les lois sont mal acceptées, mal connues, très peu diffusées et surtout peu compréhensibles pour les populations rurales en grande partie analphabètes (Le Roy, 1995). Selon Bonnet (2001), l'État, gardien de la légalité foncière, se trouve donc face à un système coutumier résistant et complexe.

Dans la Sous-préfecture de Mongo, le questionnement majeur à la base de cette recherche découle de la rareté des « bonnes » terres suite à la dégradation des conditions climatiques. On observe un accroissement de la compétition pour l'accès à la terre sur les espaces exploitables. Les activités agricoles et pastorales se disputent alors les terres, ce qui débouche le plus souvent sur des conflits entre les différents usagers. Ces conflits sont parfois exacerbés par l'action partisane des élites urbaines, dont la présence dans l'espace rural exacerbe le jeu foncier. Cette situation, si elle devait persister, pourrait hypothéquer sérieusement l'épanouissement des activités rurales, et donc le développement de cette localité. De ce questionnement découle l'hypothèse selon laquelle la persistance des conflits fonciers dans cette Sous-préfecture entraine de nombreux impacts qui constituent un facteur de blocage pour le développement rural.

\section{Méthodologique de recherche}

\subsection{Cadre d'étude}

L'étude a pour cadre la Sous-préfecture de Mongo dans ses limites fixées par le redécoupage administratif intervenu en 2002. En effet, aux termes des décrets $\mathrm{n}^{\circ}$ 415, 416, 418 et 419/PR/MAT/2002 du 17/10/02, le département du Guéra a été restructuré et deux nouvelles Sous-préfectures ont été créées à partir des cantons Migaami et Bidio (figure 1). Il s'agit respectivement des Sous-préfectures de Baro et Niergui. Ainsi, les limites territoriales de la Souspréfecture de Mongo se sont resserrées autour des cantons Dadjo I et Missirié Oyo. Toutefois, des populations issues d'autres groupes ethniques, notamment Dangléat, Bidio, Migaami s'y trouvent également. La Sous-préfecture de Mongo couvre alors une superficie d'environ $9800 \mathrm{~km}^{2}$ et une population estimée en 2009 à 73433 habitants (RGPH-2, 2009). 


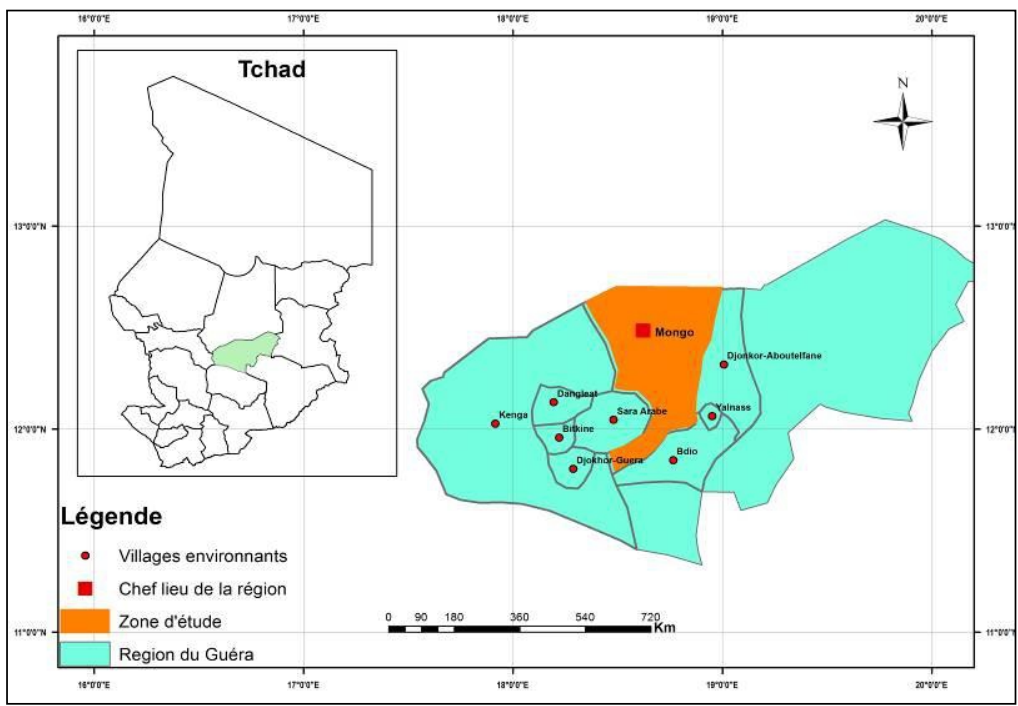

Source : CNAR, 2015.

Figure 1 : Localisation de la zone d'étude.

Il est important de relever que dans le cadre de cette étude, le travail se limite essentiellement aux zones rurales, ce qui exclut la commune urbaine de Mongo. Ainsi, on pourra constater qu'une partie d'Oyo se trouve dans les limites communales alors que le reste de villages est en pleine zone rurale.

\subsection{Collecte et traitement des données}

Deux types de données ont été nécessaires pour mener cette étude. Les données secondaires obtenues à travers la documentation disponible sur le sujet et les données primaires, collectées à partir des entretiens menés auprès des personnes ressources, et une enquête par questionnaire effectuée auprès des ménages. Un travail de pré-enquête a permis d'identifier les villages où la question foncière se pose avec le plus d'acuité. Ainsi, sur la base des informations reçues, 5 villages sur 50 et 3 férik ont été retenus pour l'enquête. Compte tenu des objectifs fixés et de l'étroitesse des moyens financiers, un échantillonnage systématique a été opéré. Les chefs des 5 villages, les cheikhs des 3 férik, l'imam de la grande mosquée de Mongo, le sous-préfet de Mongo, le chargé des programmes de l'ONG Moustagbal qui intervient dans la zone, ont été systématiquement pris en compte par l'enquête. A ces personnes ressources s'ajoute et l'élite urbaine, aujourd'hui considéré comme un nouvel acteur du jeu foncier rural. Par ailleurs, un questionnaire a été administré auprès des 106 ménages (tableau 1). 
Tableau 1 : Répartition par village/férik et par sexe des individus enquêtés

\begin{tabular}{|l|c|c|c|}
\hline \multicolumn{1}{|c|}{ Village } & Total & Hommes & Femmes \\
\hline Baiwangué & 3 & 1 & 2 \\
\hline Gourba & 7 & 3 & 4 \\
\hline Kounio & 8 & 4 & 4 \\
\hline Termel & 2 & 1 & 1 \\
\hline Tchalo-zoudou 9 & 46 & 21 & 25 \\
\hline Férik 1 & 14 & 6 & 8 \\
\hline Férik 2 & 19 & 9 & 10 \\
\hline Férik 3 & 7 & 4 & 3 \\
\hline Total & $\mathbf{1 0 6}$ & $\mathbf{4 9}$ & $\mathbf{5 7}$ \\
\hline
\end{tabular}

Source : Données d'enquête, 2014.

En ce qui concerne le traitement des données recueillies, le logiciel Excel a été principalement utilisé tandis que le logiciel Arc GIS a été nécessaire pour la réalisation de la carte de la zone d'étude. Les résultats obtenus ont permis d'organiser le travail autour de quatre points principaux à savoir l'analyse de l'évolution du cadre de la gestion foncière dans la Sous-préfecture de Mongo, l'analyse des enjeux fonciers et des conflits fonciers y afférents, l'analyse des acteurs et de leur enjeu dans la multiplication des conflits fonciers et l'impact de ces conflits sur la structuration socioéconomique du milieu rural.

\section{2. Évolution du cadre de la gestion foncière dans la Sous-préfecture de Mongo : vers l'émergence d'une pluralité d'acteurs}

\subsection{La conception traditionnelle de la terre}

Selon la coutume Hadjaray, la terre est un don de Dieu dont la gestion revient à la communauté. Sur cette base, le système coutumier local attribue la propriété de la terre aux groupes les plus étendus comme les lignages, qui se chargent de répartir les droits d'usage entre les différentes familles de la communauté. En termes clairs, seule la collectivité dispose d'un véritable droit de propriété, les individus ou les ménages ne disposent que des droits d'usage. Et l'accès de l'individu à la terre se fait au nom de son appartenance à la collectivité. Les terres ainsi attribuées sont utilisées de génération en génération par les mêmes familles qui peuvent constituer, de facto, une propriété gérée par le chef de famille, qui les attribuera à ses descendants.

Dans tous les cas, aucune valeur monétaire n'est attribuée à la terre qui se donne gracieusement. Elle ne peut faire l'objet d'une aliénation. La notion de propriété privative et individuelle n'existe pas.

\footnotetext{
${ }^{9}$ Le village Tchalo-zoudou ne fait pas partie de la sous-préfecture de Mongo, mais en raison du conflit qui l'oppose au village Kounio nous l'avons intégré dans notre zone d'étude.
} 


\subsection{Le système de gestion moderne du foncier}

L'introduction d'une réglementation foncière formelle, c'est-à-dire écrite, dans la Sous-préfecture de Mongo remonte à la période coloniale. En effet, dès son arrivée, l'administration coloniale a voulu substituer aux systèmes coutumiers une réglementation écrite qui serait conforme à la logique d'un État moderne.

La mise en place de ce système foncier est guidée par une volonté manifeste de remettre en cause toute l'architecture de règles élaborées par les communautés locales. C'est aussi la négation de toute autorité autre que celle $\mathrm{du}$ colonisateur et de toute organisation propre aux sociétés traditionnelles. Cela a désorganisé tout le jeu interne en dépossédant les notables de ce qui faisait leur prestige et leur pouvoir. Ainsi, dans la perspective d'opérer une mainmise totale sur les terres, l'autorité coloniale a édicté plusieurs textes (tableau 2).

Tableau 2 : Principaux décrets relatifs au foncier édictés par l'administration coloniale

\begin{tabular}{|c|c|c|}
\hline Année & Type de texte & Objet \\
\hline 1899 & Décret & Régime de la propriété foncière \\
\hline 1906 & Décret & $\begin{array}{c}\text { Organisation du système de l'immatriculation } \\
\text { foncière }\end{array}$ \\
\hline 1925 & Décret & Constatation des droits coutumiers \\
\hline 1932 & Décret & Immatriculation foncière \\
\hline 1935 & Décret & $\begin{array}{c}\text { Incorporation au domaine privé des terres } \\
\text { inexploitées depuis plus de dix (10) ans }\end{array}$ \\
\hline 1955 & Décret & Réorganisation foncière et domaniale \\
\hline 1956 & Décret & Application du décret du 20/05/1955 \\
\hline
\end{tabular}

Source : $C E F O D / O F T, 2004$

$\mathrm{Au}$ moment de son indépendance en 1960, le Tchad a reconduit systématiquement l'ordre juridique colonial y compris la législation foncière. Les dirigeants de l'époque n'ont pas jugé opportun de réformer les textes qui régissaient alors le foncier. Ce n'est qu'en 1967 que l'État tchadien va se doter de nouveaux textes qui n'ont d'ailleurs pas remis en cause fondamentalement les principes fonciers tels qu'édictés par l'autorité coloniale.

Tableau 3 : Lois foncières adoptées par l'État tchadien en 1967.

\begin{tabular}{|c|c|c|}
\hline Année d'adoption & Références & Objet \\
\hline \multirow{4}{*}{1967} & $\begin{array}{c}\text { Loi }{ }^{\circ} 23 / \mathrm{PR} \text { du 22 } \\
\text { juillet }\end{array}$ & $\begin{array}{c}\text { Statut des biens } \\
\text { domaniaux }\end{array}$ \\
\cline { 2 - 3 } & $\begin{array}{c}\text { Loi }{ }^{\circ} 24 / \mathrm{PR} \text { du 22 } \\
\text { juillet }\end{array}$ & $\begin{array}{c}\text { Limitations aux droits } \\
\text { fonciers }\end{array}$ \\
\cline { 2 - 3 } & $\begin{array}{c}\text { Loi n }{ }^{\circ} 25 / \mathrm{PR} \text { du 22 } \\
\text { juillet }\end{array}$ & $\begin{array}{c}\text { Propriété foncière et } \\
\text { droits coutumiers }\end{array}$ \\
\hline
\end{tabular}

Source : CEFOD/OFT, 2004.

Ces trois (03) dernières lois font de l'État tchadien le propriétaire incontesté des toutes les terres dont l'appartenance n'est, légalement, reconnue à aucune 
personne physique ou morale. Cette situation engendre un antagonisme sur les terres rurales entre l'État et les communautés villageoises, mais seulement dans le principe. En effet, malgré les droits d'usage et de jouissance qui leur sont reconnus, les populations revendiquent la pleine propriété des terres qui sont entre leurs mains. L'État tchadien n'a jamais été à la hauteur de sa prétention, et en milieu rural, les terres sont restées pratiquement sous l'emprise des règles coutumières. Cette résistance de la coutume n'a jamais été maitrisée, et aujourd'hui encore la cohabitation entre pratiques foncières traditionnelles et droit moderne est bien une réalité, entrainant de fait une pluralité des normes en matière de gestion de la terre.

\subsection{Le pluralisme des normes dans la gestion foncière}

Le pluralisme des normes est une notion d'essence juridique, mais nous avons choisi de l'utiliser ici parce qu'il traduit le mieux la situation foncière observée dans la Sous-préfecture de Mongo. En effet, en ce qui concerne notre zone d'étude, ce concept désigne la coexistence au sein de l'espace social d'une variété de normes ou règles issues de divers groupes ethniques. Il exprime une contradiction entre plusieurs systèmes de référence découlant tantôt des pratiques musulmanes, tantôt de la coutume de chaque ethnie. Chaque groupe ethnique possède, en effet, ses propres normes de gestion foncière qui définissent les modalités d'acquisition de la terre, sa transmission, ses rites agraires, le statut foncier des femmes etc. Il pose aussi le problème de l'écart entre la législation foncière en vigueur et les pratiques locales.

Cette pluralité des règles débouche sur un antagonisme entre les principes énoncés par la loi et les pratiques locales en matière d'accès à la terre (figure 1).

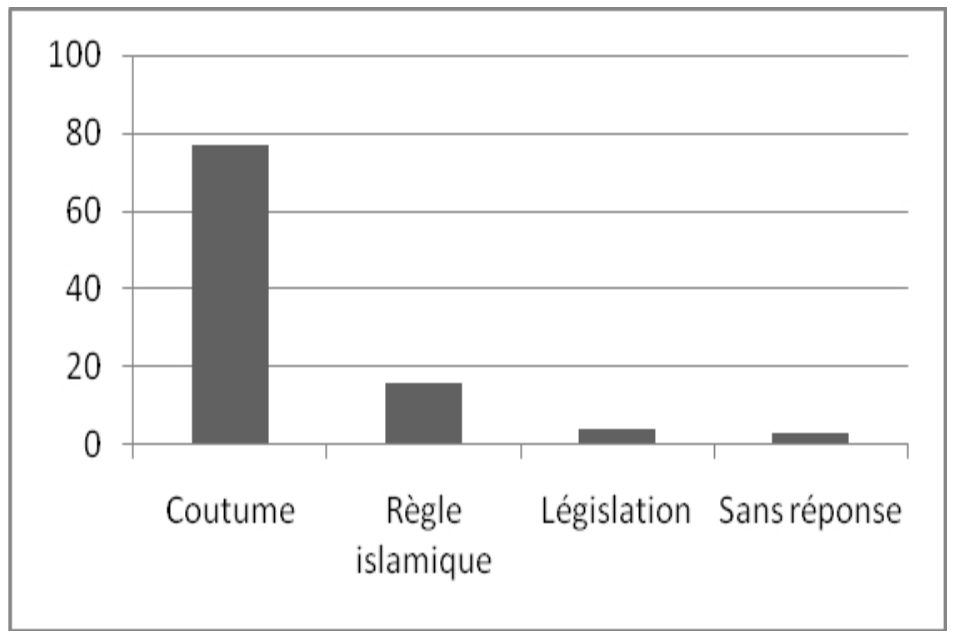

Source : Données d'enquête, 2015.

Figure 2 : La pluralité des normes foncières en usage dans la Sous-préfecture de Mongo. 
Comme on peut le voir à l'analyse de la figure 1, il existe une nette dominance de la coutume sur la législation en vigueur sur le foncier à Mongo. Deux raisons sont communément avancées pour expliquer cette tendance : la faible connaissance de la législation foncière et le paternalisme de l'État. Le choix des populations de recourir aux pratiques coutumières est aussi guidé par une logique de contestation, car elles rejettent vigoureusement l'idée que l'État garde un monopole sur les terres. Cette contradiction fondamentale entre différents systèmes de référence est en réalité l'expression d'enjeux fonciers importants face auxquels chaque acteur cherche à se positionner.

\section{De multiples enjeux fonciers dans la Sous-préfecture de Mongo}

\subsection{L'appropriation de la terre en tant qu'outil de production}

Mongo a une vocation profondément agricole et pastorale. Mais, les enquêtes de terrain montrent que l'agriculture domine car elle occupe $76 \%$ des personnes enquêtées contre moins de $20 \%$ pour l'élevage. De plus, 3 à $5 \%$ déclarent exercer comme activité secondaire le maraichage, qui est du reste à classer dans la catégorie des activités agricoles. Les principaux groupes ethniques rencontrés (Dadjo, Dangléat, Bidio, Migaami et même Arabes) pratiquent une agriculture pluviale et produisent notamment le sorgho rouge ou blanc, le millet ou pédiculaire, l'arachide, le sésame, le pois de terre, le gombo etc. La population cultive aussi le sorgho de décrue appelé «berberé » qui a un cycle plutôt tardif par rapport au calendrier agricole local (novembrejanvier). C'est une agriculture essentiellement vivrière ; seule une partie des récoltes est vendue pour se procurer certains articles (sucre, vêtements etc.) ou pour participer à certains événements sociaux dans le village (funérailles, mariage etc.). Aussi, la permanence de cette activité constitue-t-elle pour ces derniers un enjeu prioritaire à défendre.

\subsection{Enjeux pour les agriculteurs}

Du fait des conditions peu favorables, nées de la péjoration climatique de ces dernières années, l'agriculture dans la zone de Mongo est sérieusement perturbée. Les rendements agricoles baissent au fil des années à cause du déficit pluviométrique, la perte de fertilité des sols et les dégâts dus aux différents prédateurs. Par ailleurs, de plus en plus des terres sont abandonnées de façon durable à cause de leur appauvrissement car utilisées sans mise en jachère $^{10}$.

Le système agricole est demeuré archaïque et peu performant, basé sur une agriculture pluviale avec un matériel cultural rudimentaire. Plus de $88 \%$ des agriculteurs enquêtés affirment utiliser comme principal outil aratoire la houe. Seule une faible partie fait usage de la charrue (12\%). Quant au tracteur, il

${ }^{10}$ Le PASISAT (2012) estime à $20 \%$ la surface des terres abandonnées. 
demeure sous-utilisé, tant le coût de prestation est hors de portée des couches les plus pauvres qui sont aussi les plus nombreuses.

Cette situation entraine une compétition pour la terre entre agriculteurs, car les producteurs cherchent souvent à accroître les surfaces cultivées pour compenser la baisse des rendements. Pour eux, c'est la stratégie la plus accessible. Or, souvent l'extension des surfaces cultivées se traduit en réalité par une morsure sur l'espace partagé avec les éleveurs. Du fait de cette extension, l'on observe partout une diminution de la réserve en terres. Les surfaces des jachères, même de courte durée, se restreignent, passant de $50 \%$ à $25 \%$ (PASISAT, 2012) de l'ensemble des parcelles et sont de plus en plus cantonnées aux terrains les moins fertiles. Certains producteurs ont progressivement mis en culture des terres marginales peu fertiles (appelées localement «naga' $a »)$ où les rendements sont médiocres.

Dans ce contexte, on voit apparaitre de stratégies basées parfois sur l'exclusion des éleveurs. Pour les agriculteurs, l'occupation pastorale, du fait qu'elle ne laisse pas de traces pérennes, n'est pas considérée comme une forme effective de mise en valeur des terres, comparée au défrichement qui marque fortement l'occupation spatiale (Thébaut, 1995). Cela rejoint, du reste, les dispositions de la loi (art.17, loi 24) qui conditionne la mise en valeur d'une terre à une occupation permanente. D'où la précarité des droits fonciers pastoraux sur la plupart des espaces communs. C'est ce qui explique aussi la contestation d'une assise territoriale pour les éleveurs, y compris ceux du canton Misserié, toujours considérés comme des allogènes. Cette situation peu favorable au pastoralisme est liée en fait à un primat de la sédentarité sur les autres formes d'occupation du sol, et cela entretient un sérieux malentendu entre l'État et les éleveurs transhumants qui se voient ainsi lésés.

\subsection{Enjeux pour l'élevage transhumant}

Pour les éleveurs, la stratégie développée pour s'adapter à la forte variation des ressources pastorales dans le temps et dans l'espace repose sur la transhumance du bétail. Loin d'être une errance, la transhumance est un mouvement réfléchi, rythmé selon un calendrier et des itinéraires précis.

En effet, l'élevage tel que pratiqué dans la Sous-préfecture de Mongo est fortement tributaire de la disponibilité des pâturages et des points d'eau. Or, ces deux ressources sont dispersées dans l'espace et varient en quantité et en qualité dans le temps. La recherche des ces ressources pastorales impose donc une mobilité dont l'amplitude est fonction du type et de la taille du troupeau. Pour le cheptel bovin, l'essentiel des ressources fourragères est constitué de graminées annuelles à cycle court. Ainsi, chaque année, à l'approche de la saison de pluies, entre mai et juin, les éleveurs quittent les zones agricoles et commencent à remonter progressivement vers le Nord, jusqu'aux rives du Batha ou du Salamat (Nord-est). Au fur et à mesure qu'ils s'éloignent, la saison 
de pluies s'installe, et le bétail peut se nourrir et s'abreuver sans peine. La durée de séjour reste en revanche variable en fonction de la qualité de la saison, donc de la disponibilité des pâturages. A partir de septembre, alors que les mares commencent à tarir et les pâturages se raréfier, les transhumants rebroussent chemin. Vers la mi-octobre, la plupart des éleveurs atteignent les zones agricoles comme Délep, Abreche ou Banda, où ils offrent leurs services aux agriculteurs (transport des récoltes) moyennant une contrepartie en nature et profitant de la vaine pâture une fois les champs récoltés.

En dépit des sécheresses qui régulent les effectifs, le cheptel, surtout bovin, augmente très vite et l'espace pastoral se trouve ainsi saturé. Pour les éleveurs, la principale menace provient de l'extension continue des zones de cultures qui induit un rétrécissement des sites de pâturages et aggrave le déséquilibre entre la charge pastorale et les ressources disponibles. Aussi, les éleveurs mettentils en place des stratégies de marquage de l'espace dont l'objectif est de limiter l'avancée du front agricole. Par exemple à Oyo et autour du village Banda, des champs sont installés pour empêcher de nouveaux défrichements ; certains décident de créer des férik fixes sur le modèle des villages d'agriculteurs, et constituer ainsi un site d'ancrage ${ }^{11}$.

Ainsi, la terre est perçue autant par les éleveurs que les agriculteurs d'abord comme une ressource essentielle à exploiter et son contrôle relève d'une lutte pour la survie. Il n'est pas seulement question d'accéder à la terre, mais à la bonne terre et pouvoir y garder des droits durables, voire définitifs ; accéder, pour ainsi dire, à la sécurité foncière. Toutefois, si la terre est d'abord convoitée comme facteur de production par la grande partie des populations, elle est aussi utilisée comme source de pouvoir par d'autres catégories, notamment l'élite locale (autorités traditionnelles, chefs de lignage, commerçants locaux etc.).

\subsection{Le contrôle de la terre comme source de pouvoir}

$\mathrm{Au}$-delà de son importance économique, sociale et affective, la terre est également une importante source de pouvoir. En fait, contrôler la terre, c'est contrôler les moyens d'existence de ceux qui l'occupent. Cela donne au détenteur de la terre un réel pouvoir sur les utilisateurs. En effet, comme nous l'avons souligné tantôt, l'organisation sociale dans les villages est de type lignager. Les familles lignagères sont les propriétaires de la terre et les dépositaires des règles de gestion foncière. Ils fondent leur légitimité et leur autorité foncière sur le droit du premier occupant (ou droit de la hache) reconnu à leur ancêtre qui aurait mis en valeur un espace supposé non occupé.

$\mathrm{Au}$ sein des groupes lignagers, la terre est répartie entre les différents membres en fonction des besoins et de la taille de chaque ménage. Il faut relever que le système foncier traditionnel reflète les relations de pouvoir dans

\footnotetext{
${ }^{11}$ Ainsi, seule une partie de la famille va en transhumance avec le troupeau. Cette permanence
} d'occupation évite que les agriculteurs ne viennent s'installer et s'approprier l'espace pastoral. 
la société, entre les générations et entre les sexes. Ainsi, les jeunes n'ayant pas encore leur propre foyer ne peuvent avoir leur propre lopin de terre. Ils doivent, en revanche, participer à la culture du champ familial. La transmission de la terre se fait de façon héréditaire (après le décès du père) entre les enfants des deux sexes, conformément à la prescription coranique qui recommande que l'héritier mâle reçoive le double de la part de la femme ; ceci en raison de sa responsabilité sociale plus large. Par ailleurs, le code musulman réserve à la femme mariée le huitième des biens du couple en cas de décès du conjoint. Cependant, cette prescription n'est pas toujours respectée. Dans bien de cas, les proches de l'époux s'accaparent des biens, et la femme peut se voir entièrement dépossédée.

Du point de vue du droit moderne, la loi du 22 juillet 1967 régissant le régime foncier accorde le droit à tout citoyen d'accéder à la propriété foncière. Mais les droits coutumiers ne reconnaissent la propriété qu'aux hommes chefs de famille appartenant au groupe de parenté. La femme est ainsi exclue de la propriété foncière surtout en ce qui concerne les terres aménagées. Les membres du village qui ne sont pas directement rattachés à un lignage ou à une grande famille doivent défricher les terres communautaires qui constituent en quelque sorte la réserve foncière du village, et les parcelles deviennent ainsi leur propriété.

Quant aux migrants, comme les Kouka ${ }^{12}$ ou certains Arabes, ils peuvent bénéficier de la terre productive sous réserve de reconnaitre publiquement qu'elle demeure la propriété du prêteur et qu'il peut la retirer à tout moment. Cette conditionnalité signifie que le migrant est fortement déconseillé d'effectuer un investissement durable sur cette terre, par exemple planter un verger. Le prêt d'une terre donne lieu, en principe, au paiement par l'exploitant d'une sorte de redevance en nature prélevée sur sa récolte. Cette redevance constitue aussi une importante source de pouvoir économique. Toutefois, cette pratique n'est pas obligatoire, et souvent elle n'a lieu que la première année d'exploitation en signe de reconnaissance. Et l'importance du don est laissée à la stricte discrétion de l'exploitant.

Les familles lignagères désignées comme les "propriétaires » de la terre ne cultivent pas l'ensemble des domaines qu'elles détiennent et ne cherchent pas à les exploiter à leur profit exclusif. Elles sont plutôt favorables à leur distribution, à la seule condition que leur suprématie sociopolitique et leur statut de décideurs dans la répartition des terres de la collectivité soient toujours reconnus. Par contre, elles seraient très réticentes à une attribution des terres aux allogènes. La logique des détenteurs fonciers lignagers apparait alors

\footnotetext{
${ }^{12}$ Les Kouka sont originellement des habitants de la région du Batha, plus particulièrement la Sous-préfecture de Koundjourou, mais on les rencontre aussi dans certains villages dadjo, notamment à Délep et Binga.
} 
plus claire ; il s'agit de se prévaloir de leur maitrise foncière pour affirmer leur autorité et leur domination sur le village et sur les hommes.

\subsection{Le contrôle des retombées économiques et sociales}

Un des principaux enjeux qui sous-tendent les réclamations foncières c'est le contrôle des retombées économiques et sociales découlant de certaines interventions publiques ou privées dont chaque groupe cherche à profiter. En l'occurrence, le tracé et le bitumage des routes, en particulier le bitumage de la route N'Djaména-Mongo, n'y est pas étranger et explique en grande partie les tiraillements de ces dernières années dans cette zone.

En effet, c'est à travers cette grille de lecture qu'il faut analyser la plupart des conflits fonciers qui surviennent dans la Sous-préfecture de Mongo. Plus spécifiquement, nous voulons évoquer le conflit en 2012 entre Termel et Gourba au sujet de la plaine de Yarwané disputée par les deux villages. La décision de justice en faveur des premiers a suscité la fureur des seconds qui ont préféré se faire justice eux-mêmes. Le bilan fut de trois morts et plusieurs blessés suite à une farouche bataille avec arme blanche qui a opposé les deux parties devant le siège du tribunal de Mongo. L'ampleur de ce conflit a été d'autant plus surprenante que les deux parties appartiennent au même canton, donc au même groupe ethnique (Dadjo). Ce qui devrait plutôt être considéré comme un facteur d'apaisement. Puisqu'en général, le conflit est plus intense lorsque les parties adverses n'ont aucune relation de parenté.

Un autre fait illustratif est le conflit entre Dadjo et Dangléat, habitant respectivement les villages presque mitoyens de Kounio et Tchalo-zoudou. Le vendredi 24 mai 2013, un affrontement très violent a opposé les habitants des deux villages entrainant la mort de sept personnes et plus d'une trentaine de blessés. Selon les enquêtes de terrain, le problème serait parti d'une " affaire d'antenne ». En effet, la société de téléphonie mobile TIGO dans le cadre de l'extension de son réseau a décidé d'implanter une antenne de relais dans le village Tchalo-zoudou. Le site choisi pour planter le pylône se trouve être la « propriété » d'un homme appartenant à l'ethnie Dangléat ${ }^{13}$. Celui-ci devrait donc logiquement tirer tous les avantages en termes de redevances et autres découlant de cette opération. Pour les Dadjo il n'en est point question; ils estiment que c'est à eux d'en bénéficier et non aux « migrants » car il s'agit de leur terre.

Mais il est important de remarquer que "l'affaire de l'antenne " ne fut qu'un élément déclencheur. Fondamentalement, le problème trouve son origine dans la récente création de la Sous-préfecture de Bang-Bang (canton Dangléat) qui était un village voisin de Mongo. Cette érection a entrainé de facto une sorte de nouvelle légitimité pour les Dangléat sur les terres qu'ils

${ }^{13}$ En raison de leur forte imbrication, des membres des deux ethnies se trouvent de part et d'autre. 
occupaient jusque-là. Bien plus, ils estiment qu'il faut revoir les limites des deux cantons. Pour les Dadjo, en revanche, Tchalo-zoudou se trouve pleinement sur leur terre et par conséquent les Dangléat sont considérés comme des allogènes ou des personnes à statut foncier précaire. En effet, les versions sur l'antériorité de l'installation sont variables d'un groupe à l'autre. Les Dadjo affirment être les premiers occupants et qu'à l'époque les Dangléat habiteraient la montagne pour se protéger des razzias effectuées par l'empire du Ouaddaï. Par la suite, ils auraient quitté leur refuge et ont demandé à s'installer dans le voisinage des Dadjo. Et selon les bonnes manières de l'époque ceux-ci les ont accueillis en leur cédant, contre paiement d'un tribut, cette portion de terre dont ils revendiquent aujourd'hui la propriété. Les Dangléat, estiment quant à eux que la limite entre les deux cantons se situe au niveau du village Sourbodong. Ces dits sont appuyés par des faits historiques; car à l'époque l'autorité coloniale qui partait à Mongo se faisait transporter par les Dangléat qui la déposaient à Sourbodong où les Dadjo prenaient le relais, au lieu où débute leur territoire.

\section{De l'intervention d'une multitude d'acteurs dans le paysage rural à une diversité de conflits fonciers dans la Sous-préfecture de Mongo}

L'accès à la terre implique des nombreux acteurs très différents de par leurs statuts sociaux, leurs pouvoirs et leurs stratégies. Pour comprendre les paramètres qui sous-tendent les choix de chaque acteur et sur les éventuels conflits, il est indispensable de cerner le rôle de chaque acteur et l'étendue de son pouvoir.

\subsection{Les acteurs du jeu foncier dans la Sous-préfecture de Mongo}

L'échiquier foncier dans la Sous-préfecture de Mongo est essentiellement marqué par la cohabitation dans un même espace de nombreux acteurs dits ruraux (des producteurs essentiellement), auquel s'ajoute de plus en plus l'élite urbaine. Dans la catégorie de producteurs, nous mettons essentiellement les agriculteurs et les éleveurs, qui sont des usagers dont les activités s'inscrivent fortement et durablement sur le sol. Bien que leurs modes d'occupation du sol soient différents, et ce, en raison de la nature même de leurs activités respectives, la terre représente pour eux d'abord une ressource à exploiter.

En effet, il faut rappeler que la Sous-préfecture de Mongo est une zone à vocation majoritairement agricole. Néanmoins, l'agriculture pratiquée est familiale, essentiellement pluviale de type extensif, basée sur des exploitations de faibles superficies (PASISAT, 2012). Parmi ces agriculteurs, certains pratiquent également l'élevage de petits ruminants (moutons, chèvres), d'autre disposent de bovins, mais ont tendance à les confier aux pasteurs transhumants, car le cheptel bovin exige un espace plus étendu et plus ouvert. 
Les éleveurs quant à eux, ne représentent qu'une faible proportion dans la population (moins de $20 \%$ des personnes enquêtées), essentiellement constitué des transhumants et nomades qui détiennent un important cheptel dans la région.

En effet, la grande sécheresse qui a frappé la bande sahélienne dans les années 1970 et 1980 ainsi que la longue période d'instabilité que le pays a connue, ont porté un coup dur à l'élevage tchadien. Les éleveurs sont principalement issus des communautés arabes venues du Nord (Batha) ou de l'Est (Biltine) à la suite des crises écologiques successives qui ont fortement affecté leurs terroirs d'attache.

Parmi les acteurs dits ruraux, se trouve également les autorités coutumières. En raison de la plus ou moins grande homogénéité des populations, l'organisation sociale est presque la même dans tous les villages ou férik que nous avons enquêtés. La structure du pouvoir traditionnel est constante. Il existe, dans chacun de ces village un ou plusieurs chef de village, appelé « milik », selon la taille du village, ainsi qu'un Imam, qui sont les autorités de référence. Ces chefs servent d'interface avec les différents services étatiques et perçoivent certaines taxes (sur le bétail, par exemple) qu'ils reversent chez le receveur-percepteur de la circonscription. Ils s'occupent aussi du règlement des litiges entre les villageois. A côté de ces chefs, se trouvent le chef de canton, qui est un acteur de second plan, puisqu'il réside dans la ville de Mongo. Mais son influence est très grande dans la mesure où il représente l'autorité de tutelle des chefs de village ; donc il est titulaire des droits fonciers traditionnels au niveau du canton (Roasngar, 2008). Ce qui l'autorise à distribuer la terre à ceux qui en font la demande.

Aussi, aux côtés de ces autorités traditionnelles se trouve l'Imam, le guide spirituel, qui incarne au-delà une autorité morale dont l'avis est très sollicité lorsqu'il s'agit de prendre des décisions qui engagent la vie de la communauté. C'est lui qui arbitre souvent les différends et tranche les litiges y compris ceux liés à la terre. Étant donné la nature de sa fonction et l'aura de sa personnalité, ses décisions jouissent généralement d'une grande considération.

Enfin, il est important de noter que l'organisation sociale dans la Souspréfecture de Mongo est basée sur un système de lignage patrilinéaire. La succession se fait de père en fils, et le lignage regroupe toutes les personnes issues ou qui se réclament d'un ancêtre éponyme, fictif ou réel. Ainsi, dans chaque village, on peut dénombrer plusieurs lignages ou grandes familles. Chez les Dadjo et les Dangléat, il existe plusieurs clans subdivisés en grandes familles. Chaque famille est dirigée par un chef qui est son seul représentant. De même, chez les Arabes Misseriés, l'organisation sociale est de type lignager. Cette référence à l'ancêtre établit une proximité sociale entre les groupes et fait des chefs de lignage de véritables détenteurs de la terre. 
L'élite urbaine, constitue également un acteur important de la structuration de l'espace rural qui mérite d'être analysé pour comprendre comment se comportent certains acteurs face aux mécanismes d'accès à la terre. Avant tout, une clarification s'impose. Par élite urbaine, nous désignons un groupe minoritaire de ressortissants ayant des privilèges découlant soit de leur position sociale, soit des qualités intellectuelles, politiques, économiques etc. et qui, de ce fait, exercent une influence sur leur communauté d'origine. Même s'ils sont natifs, la plupart des membres de l'élite urbaine ne vivent plus au village ; ils sont soit dans la ville de Mongo soit en général à N'Djaména. Le profil de cette élite est très divers : cadres politiques, fonctionnaires, militaires, commerçants, etc. Parce qu'ils occupent des positions d'influence ou de pouvoir, les membres de l'élite maitrisent plus ou moins les jeux et les enjeux de l'accès à la terre et exercent une grande influence sur les populations villageoises. Grâce à leur capacité financière ou d'influence, l'élite urbaine est devenue un acteur important du jeu foncier dans la Sous-préfecture de Mongo. Ils peuvent ainsi acquérir d'importantes surfaces, parfois au détriment de certains exploitants locaux. D'autres par contre, préfèrent investir dans l'achat du bétail. Dans le contexte de la décentralisation, marqué par le retrait de l'État de l'échelon local au profit des collectivités locales, l'influence de l'élite urbaine s'est renforcée. Mais leur action est nécessairement à mettre en interaction avec celle des élites nationales et des autorités administratives (Magrin, 2013). Ces élites disposent de réseaux et d'appuis solides dans l'administration, leur stratégie va jusqu'à influencer le maillage territorial en faisant ériger leurs villages ou leurs férik en cantons ou Sous-préfectures ${ }^{14}$.

La cohabitation, l'intervention dans l'espace rural de l'ensemble de ces acteurs, des agriculteurs à l'élite urbaine en passant par les éleveurs est à l'origine d'un nombre important et varié de conflits fonciers.

\subsection{Typologie et manifestation des conflits fonciers dans la Sous- préfecture de Mongo}

De façon globale, dans notre zone d'étude, nous avons pu caractériser trois (03) principaux types de conflits fonciers.

- Les conflits de succession : Ces types de conflits sont sous-tendus par des logiques d'exclusion ou d'accaparement et opposent les ayants droit. Le partage d'un patrimoine foncier est une affaire très délicate qui prend plusieurs jours et peut donner lieu à des vives contestations parmi les ayants droit, surtout dans les familles polygamiques. Des conflits sont signalés également dans les cas où le père de son vivant attribue une terre à certains de ses enfants au détriment des autres. Par ailleurs certains enfants apparaissent souvent illégitimes, c'est-à-dire non reconnus par la coutume. Il s'agit notamment des

\footnotetext{
${ }^{14}$ Cas des trois nouvelles Sous-préfectures créées dans le département du Guéra.
} 
enfants issus d'une relation extraconjugale. Selon la coutume locale, ceux-ci appartiennent à leurs mères et sont donc exclus de la succession, et ce, même lorsqu'ils sont reconnus par leurs géniteurs.

- Les conflits d'appropriation: Ces types de conflit sont sous-tendus par des logiques d'accaparement de terre par certains acteurs. Deux cas se sont particulièrement révélés lors de notre enquête.

Le premier cas concerne les conflits opposant les ayants droit mineurs et leurs oncles. En général, lorsque le père décède en laissant des orphelins mineurs, leur patrimoine foncier est gardé par leurs oncles. Une fois majeurs, ces derniers peuvent réclamer leur héritage, ce qui ne se fait pas sans heurts, car dans bien de cas certains oncles ne veulent pas restituer les terres dans leur intégralité. Le conflit nait alors entre les enfants et leurs oncles, entrainant quelquefois une division dans la famille.

Le deuxième cas est relatif aux prétentions foncières des groupes voisins ou vivants sur un espace commun. Ici, la caractéristique principale des conflits est la revendication d'une même portion de terre par deux groupes ethniques ou deux communautés différentes. Le cas le plus en vue est celui des villages Kounio et Tchalo-zoudou, habités respectivement par les Dadjo et les Dangléat. Les deux villages se trouvent pratiquement sur le même terroir et ne sont séparés que par la route. Ce voisinage n'a pu empêcher un affrontement aux conséquences regrettables.

- Les conflits entre agriculteurs et éleveurs : Dans la Sous-préfecture de Mongo, comme partout dans le reste du pays, agriculteurs et éleveurs partagent les mêmes espaces géographiques. Dans presque tous les villages, on peut remarquer la présence d'éleveurs, soit de passage pour abreuver leur troupeau au puits villageois ${ }^{15}$, soit campant non loin des zones de culture. Agriculteurs et éleveurs utilisent les mêmes espaces quoique les systèmes de production, les catégories spatiales soient différents. En fonction de ses besoins, chaque groupe met en place ses règles en vue de maitriser son cadre écologique (Reounodji, 2004). La superposition des deux usages différents sur le même espace est à la base du contentieux entre les deux communautés. En effet, pour les agriculteurs l'espace fait l'objet de droits individualisés ou collectifs inviolables. En revanche, pour les éleveurs l'espace est ouvert et personne ne peut avoir le droit d'exclure l'autre. Cette situation ambiguë est en réalité née de l'absence d'un zonage qui définit des aires claires pour chacune des activités. La loi $\mathrm{n}^{\circ} 04$ d'octobre 1959 qui devait réglementer les conditions de transhumance est depuis longtemps tombée en désuétude. La seule solution trouvée jusqu'à présent est le balisage des pistes de transhumance qui est censé permettre la canalisation des troupeaux suivant un itinéraire convenu, en principe, par les différents acteurs.

${ }^{15}$ La loi islamique considère l'eau de surface comme un don de Dieu dont l'accès ne doit faire l'objet d'aucune restriction ou servitude, ni pour les hommes ni pour le bétail. 
Comme on peut le constater, les conflits entre agriculteurs et éleveur sont de loin les plus importants et opposent deux logiques d'occupation de l'espace. Ces conflits sont signalés dans tous les villages enquêtés à la fois par les agriculteurs, les éleveurs ainsi que par les autorités (Sous-préfet de Mongo, chef de canton). Ils sont essentiellement dus aux dégâts causés par le bétail sur les cultures. Ces dégâts vont de la destruction de quelques cultures à la dévastation des champs entiers. En 2013 par exemple, 25 champs de sorgho ont été dévastés au village Gourba. D'après les agriculteurs, les destructions de cultures sont pour la plupart les conséquences du manque de vigilance des bergers trop jeunes, ou débordés par le nombre élevé du cheptel. Aussi, le long de la plupart de pistes il n'est prévu ni point d'abreuvement, ni site de repos pour les hommes et le bétail. De ce fait, les pasteurs, fatigués, laissent le troupeau divaguer, occasionnant des dégâts sur les cultures.

Il est important de noter que sur plusieurs sites, nous avons constaté que les couloirs de transhumance qui ont été matérialisés pour limiter les conflits entre éleveurs et agriculteurs ne sont pas respectés, agriculteurs et éleveurs se rejettent la responsabilité. Les activités se chevauchent dans le temps, créant un malentendu permanent. Souvent, les agriculteurs débordent sciemment les limites fixées, envahissent les balises et, de ce fait, rétrécissent les couloirs. Cela est particulièrement remarqué dans les villages Délep et Gourba (photo 1).

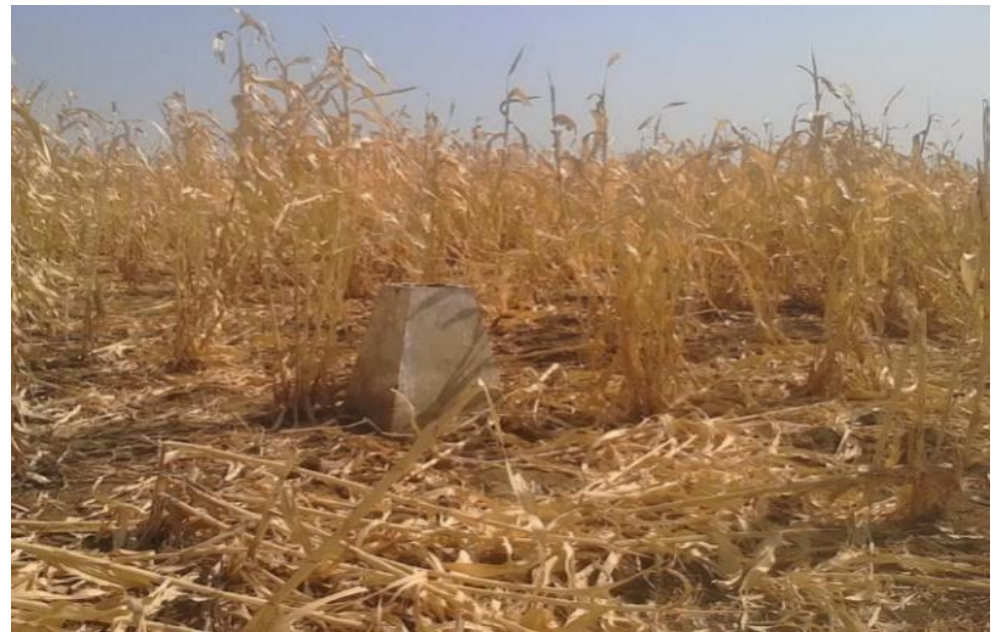

Source : Enquête de terrain, 2014.

Photo 1 : Une balise complètement envahie par un champ à Délep.

Comme on peut le constater, les champs débordent largement les couloirs de transhumance, ce qui rend floues les limites fixées pour les deux activités et contraste avec les objectifs du projet Tchad-central qui, afin de limiter les conflits entre agriculteurs et éleveurs, a balisé différents itinéraires à l'aide de ces bornes en béton, disposées de part et d'autre des pistes. Interrogés à ce 
sujet, plus de la majorité des éleveurs (63\%) estiment que c'est par pure provocation que les agriculteurs étendent leurs champs de façon anarchique au delà des limites fixées. A l'analyse, l'opération d'implantation des balises est souvent mal réalisée. Les entreprises chargées de les réaliser n'appréhendent pas vraiment les enjeux. Elles n'associent pas suffisamment les principaux acteurs concernés, à savoir les agriculteurs et les éleveurs.

En effet, ces dernières années certaines autorités administratives (gouverneur, préfet), militaires et élites investissent dans l'élevage et confient la garde de leur bétail aux transhumants. Ceux-ci ont alors la facilité d'avoir des armes qu'ils n'hésitent pas à utiliser lors d'affrontement avec les agriculteurs. Parfois, ce sont d'anciens combattants qui sont employés et qui ne respectent ni réglementation, ni pratique sociale (ICG, 2011). On est dans un contexte d'impunité qui les pousse parfois à laisser délibérément le troupeau entrer dans certains champs.

L'une des causes des conflits est à chercher dans le redécoupage territorial inapproprié. En effet, jusqu'à la fin des années 1980, le Tchad était un État unitaire centralisé avec un pouvoir fort. Le pays était organisé en 14 préfectures dirigées par des cadres administratifs, les préfets, nommés directement par l'exécutif. La Constitution de la République du Tchad adoptée le 31 mars 1996 et révisée en 2005 consacre la décentralisation comme mode de gestion administrative du territoire et définit 4 niveaux de collectivités territoriales décentralisées (CTD) : les communautés rurales, les communes, les départements et les régions. Les régions, les départements et les communes ont été créés en 2003 par l'Ordonnance n 01/PR/2003. Ainsi, en dehors de la ville de N'Djaména qui dispose d'un statut particulier, 17 régions, 47 départements et 199 communes ont vu le jour. Le 19 février 2008, le nombre des régions est passé à $22^{16}$ et depuis le 04 septembre 2012 à 23 .

Au niveau de notre zone d'étude, cette prolifération d'unités administratives a entrainé un bouleversement majeur. Mongo qui était le chef-lieu de la préfecture du Guéra garde son statut par rapport à la région nouvellement créée, mais voit son territoire morcelé au profit de 2 nouvelles Sous-préfectures qui étaient jusque là des simples villages, chefs-lieux de canton : il s'agit de Baro et Niergui. Cependant, les ressorts territoriaux et les limites géographiques de ces deux nouvelles entités ne sont pas formellement définis par rapport à la Sous-préfecture de Mongo. Pour certains, il faut alors se référer aux limites des différents cantons à partir desquels ont été créées ces nouvelles unités. Celles-ci ont été fixées pour l'essentiel pendant l'époque coloniale et les documents qui en attestent sont soit inexistants, soit récusés faute de crédibilité. Cette situation donne l'occasion aux populations de proclamer elles-mêmes les limites de leurs terroirs, faisant appel alors à des vagues

${ }^{16}$ Voir l'ordonnance $\mathrm{n}^{\circ}$ 002/PR/08 portant restructuration de certaines collectivités territoriales décentralisées. 
souvenirs et à une interprétation partiale de l'histoire. Cela constitue une source permanente de contestation entre les acteurs locaux. Avec cette érection, des villages voisins qui étaient jusqu'alors en bons termes se retrouvent dans deux Sous-préfectures différentes et se mettent à se disputer les terres sur lesquelles ils cultivaient autrefois en paix. C'est dans cette perspective qu'il faut situer à titre d'exemple le conflit entre Kounio et TchaloZoudou ainsi que celui entre Roumou et Kilgnata.

La décentralisation se retrouve ainsi déviée de son objectif initial, à savoir la dévolution des compétences et des moyens aux échelons appropriés pour mieux faire participer les populations locales à la gestion publique. En effet, le découpage territorial est utilisé par les élites politiques de la région pour promouvoir leurs villages sans aucune considération d'efficience ou d'efficacité. Il s'agit pour eux de démontrer leur envergure politique et leur poids aux yeux de leur communauté. Cette situation a favorisé l'émergence de stratégies de positionnement et d'anticipation autour de la gestion du pouvoir local et des ressources foncières. Un esprit de compétition s'est instauré parmi ces acteurs : chacun voulant avoir son canton, sa Sous-préfecture, sa région etc. De même, le pouvoir dans une logique électoraliste, est constamment à la recherche de la maille territoriale adéquate (Magrin, 2013), ce qui se traduit sur le terrain par un remodelage permanent de la configuration générale du territoire (des cantons jusqu'aux régions). Dans la Sous-préfecture de Mongo, le resserrement de la maille territoriale s'est accompagné d'une nette montée de rivalités autour de la terre et des revendications identitaires entre des groupes qui vivaient jusque-là en parfaite harmonie.

Si les conflits fonciers les plus graves en termes d'impact socioéconomique opposent des groupes ou des communautés, il n'en demeure pas moins que la grande partie ne concerne que les individus qui représentent $78 \%$ de cas de conflits, contre seulement $8 \%$ de cas de conflits collectifs (figure 3 ).

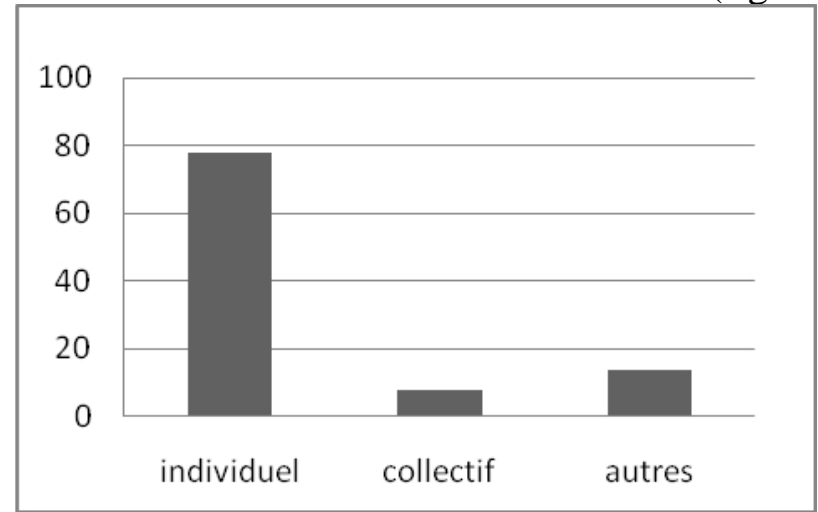

Source: Données d'enquête, 2014.

Figure 3. Typologie des conflits dans la Sous-préfecture de Mongo. 
Sur un total de 63 cas de conflits recensés, 49 ont opposé des individus entre eux au sujet des limites des parcelles ou de la divagation du bétail dans les champs, contre seulement 5 ayant engagé des groupes, voire tout un village. Des conflits dont les conséquences sur le plan socioéconomique sont remarquables.

\subsection{Impact des conflits fonciers sur le développement en milieu rural}

La terre est devenue à Mongo un enjeu capital. Les conflits pour son accès et son contrôle se multiplient, entrainant des conséquences considérables sur les composantes essentielles de la vie rurale. Les impacts de ces conflits se déclinent en termes de paupérisation des ménages, perturbation des échanges économiques entre les communautés impliquées et de crise de confiance.

\subsubsection{La paupérisation des ménages}

Les conflits fonciers entrainent d'importants dommages qui affectent plusieurs composantes socioéconomiques et sapent la dynamique de développement des zones rurales. Les dévastations de champs ou les tueries de bétail signifient une perte des moyens d'existence de la famille, contraignant les paysans à rechercher de nouvelles solutions de survie. La solution la plus courue est l'emprunt d'argent ou de vivres auprès du groupement villageois remboursable à la prochaine récolte. Dans le cas où celle-ci n'est pas possible, ces derniers sont obligés de recourir au prêt usuraire. Selon les enquêtes de terrain, 75\% de paysans affectés par la destruction des cultures ou des troupeaux, recourent à l'emprunt d'argent ou de vivres auprès du groupement villageois alors que $25 \%$ recourent au prêt usuraire, passant ainsi des situations de stabilité à une instabilité financière de ménage. Dans un milieu où l'essentiel des ressources alimentaires est tirée des champs, la destruction des récoltes constitue un coup dur pour son propriétaire. Cette situation est d'autant plus difficile que le pouvoir d'achat des agriculteurs est faible. En plus, il faut en moyenne 4 à 5 mois de dur labeur pour obtenir 5 à 10 sacs de sorgho, principale céréale consommée dans la zone. Une partie de la récolte est ensuite utilisée pour rembourser les emprunts d'argent ou de vivres contractés.

De la même manière que les champs sont détruits, le bétail fait l'objet de tuerie ou de blessures graves.

Tableau 4 : Nombre d'animaux tués par espèce dans quelques villages entre 2011 et 2014

\begin{tabular}{|l|c|c|c|c|}
\hline \multicolumn{1}{|c|}{ Villages } & Boufs & Moutons/chèvres & Dromadaires & Anes \\
\hline Gourba & 3 & 1 & 0 & 1 \\
\hline Oyo & 3 & 4 & 0 & 1 \\
\hline Tchalo-Zoudou & 4 & 2 & 1 & 1 \\
\hline Termel & 2 & 0 & 0 & 2 \\
\hline Total & $\mathbf{1 2}$ & $\mathbf{7}$ & $\mathbf{1}$ & $\mathbf{5}$ \\
\hline \multicolumn{5}{r}{ Source : Données d'enquête, 2015. }
\end{tabular}


Par ailleurs, la reconstruction des habitations détruites lors des conflits se fait au prix d'énormes efforts. Étant donné le caractère prioritaire du logement, les biens du ménage (récoltes, bétail) sont mobilisés et convertis en valeur pour l'achat des matériaux nécessaires. Cet investissement se fait souvent au détriment d'autres priorités telles que la scolarité des enfants. Pour rappel, suite au conflit qui a opposé les villages Tchalo-Zoudou et Kounio, le Gouverneur de la région du Guéra a ordonné la démolition au bulldozer de 35 cases construites sur l'espace litigieux. Une situation qui annihile les efforts réalisés et enferme le paysan dans un cercle vicieux de la pauvreté.

\subsubsection{Des échanges fortement perturbés}

Les conflits fonciers portent également un coup aux échanges entre les groupes ou communautés impliquées. En particulier la fréquentation des marchés hebdomadaires se trouve au premier plan. Par exemple, au lendemain du conflit entre les villages Termel et Gourba au sujet de la plaine de Yarwané, les premiers ont boycotté pendant plusieurs mois, voire définitivement pour certains, le marché de Banda qui se tient tous les jeudis. Cette situation n'a certes pas empêché le marché de fonctionner convenablement puisque les gens viennent de plusieurs autres villages et même de la ville de Mongo. Cependant, elle a réduit le volume de certains échanges spécifiques et la circulation des biens entre les deux villages.

\subsubsection{Une rupture de confiance entre les acteurs}

L'un des principaux impacts des conflits fonciers se traduit par le relâchement des rapports sociaux. En effet, comme nous l'avons constaté, au lendemain des conflits fonciers survenus entre les différents groupes dans la Sous-préfecture de Mongo, des traces persistent. Même dans le cas où le contentieux trouve un arrangement accepté par les différentes parties en conflit, les relations restent toujours crispées. Lorsque le conflit se solde par des cas de morts d'hommes, le fil peut être complètement rompu entre les acteurs impliqués : mariages gelés, épouses répudiées, récupération d'objets confiés ou de terres prêtées. La confiance est alors perdue et les deux camps ou communautés vivent dans la peur et la méfiance réciproque. Ceci peut parfois perdurer pendant plusieurs générations.

\subsubsection{Perturbation du calendrier cultural, insécurité et augmentation des dépenses}

Les conflits fonciers surviennent généralement durant la période des travaux dans les champs. D'où leur caractère perturbateur pour les activités agricoles et pastorales. Ceci entraine de grandes pertes pour les acteurs en conflit. 
En effet, c'est pendant les semis qu'éclatent souvent les premiers accrochages, tant entre agriculteurs et éleveurs qu'entre agriculteurs euxmêmes. Une bonne partie du temps qui aurait dû être utilisée avec profit dans le labour du champ ou de l'entretien du bétail est alors perdue. Certaines parcelles doivent parfois être ressemées, ce qui nécessite l'achat de nouvelles semences, donc de nouvelles dépenses. Parfois, le risque est grand de voir toute l'année gâchée, étant donné la brièveté de la saison de pluies dans la zone sahélienne.

Il est rare, voire inhabituel, qu'un conflit foncier s'éteigne au lendemain d'un affrontement. En général, les premiers affrontements sont considérés par les deux camps comme une sommation, un avertissement pour des batailles à venir éventuellement plus violentes. Ainsi, les populations sont toujours sur leur garde, craignant un possible assaut de l'adversaire.

Dans le cas du conflit entre les villages Tchalo et Kounio, armés de lances, haches, coutelas, gourdins etc., les hommes montent la garde autour du village afin de signaler tout mouvement suspect. Pendant des jours, voire des semaines, les travaux de champ passent ainsi au second rang des priorités. Même les femmes n'y vont que de façon irrégulière. Entre temps les mauvaises herbes envahissent les champs, étouffent les cultures. Lorsqu'enfin la tension tombe et que les paysans reprennent le chemin des champs, certaines cultures comme l'arachide, le sésame ou le gombo sont complètement étranglées. Quant au sorgho, plus résistant, il peut se relancer dès qu'il est débarrassé des mauvaises herbes, mais ne donne plus le rendement escompté. Dans les zones hydromorphes, les paysans peuvent compter sur le sorgho de décrue qui est, d'ailleurs, conditionné par la qualité de la saison.

Lorsque l'affaire est portée devant la justice, les démarches mobilisent la communauté tout entière. En particulier lorsque le conflit se solde par des cas de meurtre, c'est en masse que les membres de la communauté doivent se déplacer pour assister aux audiences du tribunal. Dans ce cas la probabilité d'une attaque par le camp adverse, pour venger ses morts ou contester une décision rendue, est très élevée. C'est exactement ce qui s'est passé quand les villageois de Termel ont été attaqués par ceux de Gourba, au sortir même de l'audience Les gens vivent donc avec la hantise d'une insécurité permanente.

\section{Conclusion}

$\mathrm{Au}$ terme de cette analyse, l'hypothèse que nous avons formulée au départ de cette recherche se trouve confirmée, à savoir que les conflits fonciers en milieu rural constituent un véritable facteur limitant le développement des campagnes. Dans un milieu où le plus grand nombre dépend de la terre pour ses besoins alimentaires, cette situation est d'une extrême gravité.

En effet, la gestion de la terre et des ressources qu'elle porte est fondée essentiellement sur des règles coutumières, religieuses et juridiques qui font 
d'elle, selon le cas, un patrimoine communautaire ou un bien cessible. Dès lors, une forte compétition pour l'accès à cette ressource s'instaure entrainant nombre de conflits. De façon générale, trois principaux types de conflits fonciers se dégagent avec en toile de fond l'accès et le contrôle de la ressource au détriment des autres. Il s'agit notamment des conflits de succession, des conflits d'appropriation et des conflits entre agriculteurs et agriculteurséleveurs. Ils peuvent soit impliquer des individus isolés, soit prendre une dimension communautaire.

Les facteurs explicatifs des conflits sont très divers, mais aucun ne peut s'imposer de façon déterminante. En revanche, parmi les enjeux, il y a la volonté de contrôler les avantages économiques ou sociaux issus de certaines interventions publiques, le désir de positionnement politique pour les élites, la reprise des terres données ou prêtées à d'autres groupes. La multiplicité des groupes ethniques vivant sur des terroirs communs ou voisins constitue de ce point de vue une véritable bombe à retardement que la moindre étincelle ferait exploser. Quant aux modes de résolution, ils sont aussi diversifiés. En dehors des instances formelles, les acteurs locaux ont mis en place des structures et des mécanismes propres qui leur permettent de gérer les litiges qui naissent des différends fonciers. Il s'agit en réalité d'alternatives à un système judiciaire devenu inopérant. Les conflits fonciers impliquent des conséquences énormes sur les dynamiques de développement des populations, dans la mesure où ils affectent des composantes essentielles de leur vie économique et sociale (production, échanges, solidarité, sécurité). Aussi, pouvons-nous affirmer, une fois de plus, qu'il y a une causalité évidente entre les conflits fonciers et le retard de développement des zones rurales.

$\mathrm{Au}$-delà de cette problématique fondamentale, il parait légitime de s'interroger sur le devenir même de la question foncière qui est en train de s'imposer comme l'une des conditions, sinon la seule, pour asseoir un développement socioéconomique harmonieux et durable. En raison de sa capacité à susciter les convoitises et à cristalliser les antagonismes, nous sommes tentés de nous interroger si la ruée vers la terre ne faisait pas que commencer?

\section{References:}

1. Bonnet, B., 2001. Problématique foncière et gestion des ressources communes. IRAM, Porto Alègre, $8 \mathrm{p}$.

2. CEFOD/OFT., 2004. La question foncière au Tchad. Actes du colloque scientifique de N'Djaména, 28 juin-1er juillet. Ed. IDT. 249 p.

3. LeRoy, E., 1995. La sécurisation foncière dans un contexte africain de marchandisation imparfaite de la terre in «Dynamiques des systèmes agraires. Terre, terroir, territoire. Les tensions foncières ». Pp 455-472. 
4. LeRoy E., Durand-Lasserve A., 2012. La situation foncière en Afrique à l'horizon 2050. Comité technique Foncier et Développement, AFD. $155 \mathrm{p}$.

5. Houdeingar D., 2009. L'accès à la terre en Afrique subsaharienne in "L'accès à la terre et ses usages: variations internationales", Rencontres Lascaux, Nantes, France. 21 p.

6. ICG. 2011. «Le Nord-ouest du Tchad: la prochaine zone à haut risque ?» 17 février. $\mathrm{Pp}$ 8-9.

7. Magrin, G., 2013. Voyage en Afrique rentière. Une lecture géographique des trajectoires du développement. Publications de la Sorbonne. $417 \mathrm{p}$.

8. Reounodji, F., 2004. Situation foncière pastorale et perspectives de sécurisation des systèmes pastoraux au Tchad in «La question foncière au Tchad». Actes du colloque scientifique de N'Djaména, juin-juillet 2004.

9. PASISAT., 2012. Bulletin d'information sur la sécurité alimentaire $\mathrm{n}^{\circ}$ 5 , région du Guéra. Octobre. $12 \mathrm{p}$.

10. RGPH-2., 2009. Recensement général de la population et de l'habitat. Résultats globaux définitifs. INSEED. $155 \mathrm{p}$.

11. Roasngar T. A., 2008. L'accès à la terre au Tchad. Ed. IDT, coll. « Le droit pour tous $\gg .87 \mathrm{p}$.

12. Thébaut, B., 1995. Le foncier dans le sahel pastoral: situation et perspectives in "Dynamiques des systèmes agraires. Terre, terroir, territoire. Les tensions foncières ». Pp 37-56. 